\title{
Estudo reológico comparativo entre duas variedades de argilas esmectitas do município de Boa Vista no estado da Paraíba
}

Comparative rheological study between two varieties of smectite clays from the municipality of Boa Vista in the state of Paraíba

Estudio reológico comparativo entre dos variedades de arcillas esmectitas del municipio de Boa

Vista en el estado de Paraíba

Recebido: 08/12/2021 | Revisado: 12/12/2021 | Aceito: 17/12/2021 | Publicado: 02/01/2022

Jéssica Guimarães Barros ORCID: https://orcid.org/0000-0001-6103-2151 Universidade Federal de Campina Grande, Brasil E-mail: jessicaguimaraes89@gmail.com

Diego José Araújo Bandeira ORCID: https://orcid.org/0000-0002-6245-9875 Universidade Federal de Campina Grande, Brasil

E-mail: diegoimperium8@gmail.com

Eduardo Sérgio Soares Sousa

ORCID: https://orcid.org/0000-0003-0893-5305

Universidade Federal de Campina Grande, Brasil E-mail: esergiosousa@uol.com.br

Lívia de Almeida Rocha

ORCID: https://orcid.org/0000-0002-7494-2530 Centro Universitário UNIFACISA, Brasil E-mail: livia.arqui@hotmail.com

Jonildo Cavalcanti da Silva Filho ORCID: https://orcid.org/0000-0001-5119-8377 Universidade Federal de Campina Grande, Brasil E-mail: jonildofilho@gmail.com

Patrício Gomes Leite

ORCID: https://orcid.org/0000-0001-5500-7865 Universidade Federal do Maranhão, Brasil E-mail: pgomesleite@gmail.com

Antônio Nunes de Oliveira ORCID: https://orcid.org/0000-0001-5697-8110 Instituto Federal do Ceará, Brasil E-mail: nunes.vieira@ifce.edu.br

Marcia Janiele Nunes da Cunha Lima ORCID: https://orcid.org/0000-0003-3697-8724 Universidade Federal de Campina Grande, Brasil E-mail: marciacunhalima87@ hotmail.com

Ariadne Soares Meira

ORCID: https://orcid.org/0000-0001-8740-9009 Universidade Federal de Campina Grande, Brasil E-mail: ariadnesm_eng@hotmail.com

Keviane Pereira Aragão ORCID: https://orcid.org/0000-0002-2237-130X Universidade Federal de Campina Grande, Brasil E-mail: kevianearagao@gmail.com

\section{Resumo}

Devido as suas propriedades, as argilas bentonitas apresentam uma vasta gama de aplicações, inclusive no setor industrial. Uma importante aplicação das argilas acontece na indústria petrolífera. O Estado da Paraíba possui uma grande variedade dessas argilas, porém, a exploração em grande escala acarretou uma diminuição em sua quantidade, o que causou um grande impacto na qualidade das mesmas. Desta forma, o objetivo desse trabalho é comparar duas argilas oriundas da Paraíba, do município de Boa Vista, denominadas Bofe e Chocolate, para uso em fluidos de perfuração de base água. Ambas as argilas foram caracterizadas por análise granulométrica (AG), análise química por fluorescência de raios $\mathrm{x}$ (EDX), difração de raios $\mathrm{x}$ (DRX), análise térmica diferencial e termogravimétrica (DTA e TG) e capacidade de troca de cátions (CTC). As propriedades reológicas foram determinadas através das medidas da viscosidade aparente, viscosidade plástica e volume de filtrado. As caracterizações comprovaram que as amostras se 
referem a argilas bentoniticas, além de apresentarem as características das argilas típicas do estado da Paraíba. Após o estudo comparativo entre as duas argilas concluiu-se que apenas a argila Chocolate apresenta valores de viscosidade aparente e volume de filtrado de acordo com as normas da Petrobrás, enquanto que a argila Bofe atende apenas as especificações de volume de filtrado.

Palavras-chave: Argilas; Bentonitas; Propriedades reológicas.

\begin{abstract}
Due to its properties, bentonite clays have a wide range of applications, including in the industrial sector. An important application of clays takes place in the oil industry. The State of Paraíba has a great variety of these clays, however, the large-scale exploration resulted in a decrease in their quantity, which caused a great impact on their quality. Thus, the objective of this work is to compare two clays from Paraíba, in the municipality of Boa Vista, called Bofe and Chocolate, for use in water-based drilling fluids. Both clays were characterized by particle size analysis (AG), chemical analysis by x-ray fluorescence (EDX), x-ray diffraction (DRX), differential thermal and thermogravimetric analysis (DTA and TG) and cation exchange capacity (CTC) . Rheological properties were determined by measuring apparent viscosity, plastic viscosity and filtrate volume. The characterizations proved that the samples refer to bentonitic clays, in addition to presenting the characteristics of typical clays from the state of Paraíba. After a comparative study between the two clays, it was concluded that only Chocolate clay presents values of apparent viscosity and filtrate volume in accordance with Petrobras standards, while Bofe clay only meets the specifications of filtrate volume.
\end{abstract}

Keywords: Clays; Bentonites; Rheological properties.

\title{
Resumen
}

Por sus propiedades, las arcillas bentoníticas tienen una amplia gama de aplicaciones, incluso en el sector industrial. Una aplicación importante de las arcillas tiene lugar en la industria petrolera. El Estado de Paraíba tiene una gran variedad de estas arcillas, sin embargo, la exploración a gran escala resultó en una disminución en su cantidad, lo que provocó un gran impacto en su calidad. Así, el objetivo de este trabajo es comparar dos arcillas de Paraíba, en el municipio de Boa Vista, denominadas Bofe y Chocolate, para su uso en fluidos de perforación a base de agua. Ambas arcillas se caracterizaron por análisis de tamaño de partícula (AG), análisis químico por fluorescencia de rayos X (EDX), difracción de rayos X (DRX), análisis diferencial térmico y termogravimétrico (DTA y TG) y capacidad de intercambio catiónico (CTC). Las propiedades reológicas se determinaron midiendo la viscosidad aparente, la viscosidad plástica y el volumen de filtrado. Las caracterizaciones demostraron que las muestras se refieren a arcillas bentoníticas, además de presentar las características de arcillas típicas del estado de Paraíba. Luego de un estudio comparativo entre las dos arcillas, se concluyó que solo la arcilla Chocolate presenta valores de viscosidad aparente y volumen de filtrado de acuerdo con los estándares de Petrobras, mientras que la arcilla Bofe solo cumple con las especificaciones de volumen de filtrado.

Palabras clave: Arcillas; Bentonitas; Propiedades reológicas.

\section{Introdução}

No Brasil são encontrados inúmeros jazimentos de argilas bentoníticas, tendo como destaque o Estado da Paraíba. Estudos apontam que em 2014 cerca de 79,3\% dessas argilas foi fornecido pelo Município de Boa Vista no Estado da Paraíba (Lima \& Neves, 2014.)

Os primeiros estudos da argila bentonita ocorreram em 1898 a um tipo de argila plástica coloidal encontrado em Fort Benton em Wyoming (EUA), que apresentava a interessante característica de aumentar várias vezes o seu volume quando umedecida em água (Santos, 1992).

Define-se por argila bentonítica, qualquer argila que contém de forma predominante o argilomineral Montmorilonita, pertencente ao grupo das esmectitas. Bentonitas são rochas que apresentam como componente predominante uma esmectita mineral gerada através da alteração de materiais vítreos de origem ignea. Elas possuem grande versatilidade, baixo custo e existem em abundância, por isso a sua importância no mundo tecnológico (Grim \& Guven, 1978; Bergaya et al, 2006; Murray, 2007; Vieira et al., 2021).

Os principais fatores que irão interferir na formação da bentonita são o clima, a vegetação e a topografia (Abdou et al, 2013). Atualmente, o mercado apresenta maior concentração nos EUA, que é considerado o maior produtor mundial. No Brasil, os principais depósitos encontram-se na Paraíba, São Paulo, Bahia e Piauí (Pereira et al., 2015).

A bentonita natural e de baixo custo é muito utilizada para a remoção de contaminantes (Toor, 2012). Tendo como 
principal composto a montmorrilonita 2:1, uma estrutura em camadas. É vista como um material que apresenta uma boa absorção, devido a sua área superficial, e características como uma grande capacidade de adsorção e a sua capacidade de inchamento.

Os minerais presentes nas argilas são considerados não tóxicos a seres humanos e animais, e são frequentemente utilizados em cosméticos e fármacos (Lee et al., 2005; Liu et al, 2011; Corrales et al., 2012). As propriedades físicas e químicas dos argilominerais do grupo das esmectitas são controladas basicamente por tamanho de partícula, formato de partícula, substituições na rede cristalina e área superficial (Rodrigues et al., 2020). Na construção civil a argila é utilizada para confecção de blocos, pisos, tubos e telhas cerâmicas bem como para fabricação de utensílios domésticos como cubas de pias ou lavabos, aparelhos sanitários e demais objetos aplicados à decoração do imóvel (Oliveira et al., 2021; Silva \& Pereira, 2021).

Como fluidos de perfuração, elas ganham destaque e movimentam centenas de milhões de dólares no mercado mundial. Esses fluidos são de extrema importância durante toda a operação de perfuração (Nascimento et al., 2010). Devido a essa importância, muitas pesquisas são realizadas quanto á caracterização desse material, com o objetivo de conhecer sua estrutura e suas principais propriedades.

O comportamento reológico das argilas bentonitas também lhes permitem outras aplicações industriais como corantes, indústria farmacêutica (Cara et al, 2000a, Cara et al, 2000b; Viseras et al, 2010), indústria de papel, cimento, nano compósitos (Araujo et al, 2004) e compósitos poliméricos (Abu-Jdayil et al, 2011).

Na perfuração de poços de petróleo existem requisições ambientais que se tornam cada vez mais rígidas, tornando assim o seu estudo mais frequente para buscar novos desenvolvimentos para fluidos a base água. Visto que os mesmos se encontram em crescente utilização e estudo (Nascimento et al., 2010, Thomas, 2001).

De acordo com a norma (EP-1EP-00011-A, 2011) da Petrobrás, para serem aplicadas em fluidos de perfuração, as argilas bentonitas devem apresentar algumas características, dentre elas apresentar viscosidade aparente igual ou superior a 15 centipoise e volume de filtrado igual ou inferior a 18 milímetros.

O trabalho teve como objetivo realizar um estudo comparativo entre dois tipos de argilas bentonitas do Município Boa Vista, todas do Estado da Paraíba, visando seus usos em fluidos de perfuração de poços de petróleo base água.

\section{Metodologia}

Para o desenvolvimento deste trabalho foram utilizadas amostras de argilas bentonitas naturais extraída de vários depósitos do Estado da Paraíba, no Munícipio de Boa Vista.

A mistura das argilas foi obtida utilizando-se a técnica de planejamento experimental de misturas, sendo denominadas de AM3 e AM4 para as variedades Chocolate e Bofe, conforme descrito a seguir:

Argila Bentonita AM3 - Argila bentonita denominada de chocolate, cedida pela Bentonita União Nordeste S.A., situada no Município de Boa Vista/PB;

Argila Bentonita AM4- Argila bentonita denominada de bofe, cedida pela Bentonita União Nordeste S.A., situada no Município de Boa Vista/PB.

$\mathrm{O}$ aditivo utilizado para transformar as argilas policatiônicas em sódicas foi o carbonato de sódio ( $\mathrm{Na2 \textrm {CO }})$ anidro PA, fabricado pela Vetec. Todos os ensaios de caracterização foram realizados no Laboratório de Tecnologia de Materiais e no Laboratório de Caracterização de Materiais da Unidade Acadêmica de Engenharia de Materiais da Universidade Federal de Campina Grande.

As etapas desenvolvidas deste trabalho foram executadas obedecendo ao fluxograma representado na Figura 1. 
Figura 1. Fluxograma das etapas executadas no projeto.

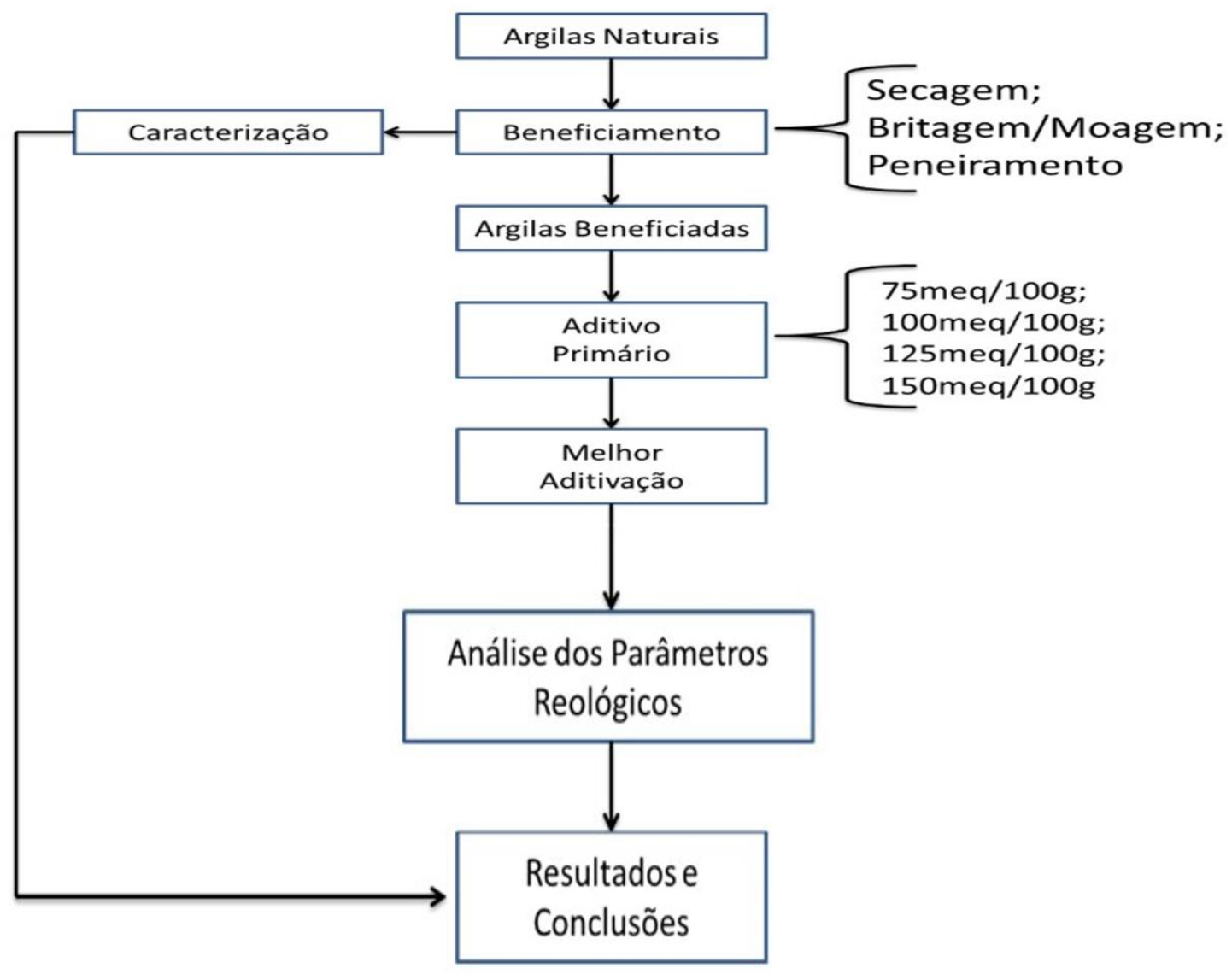

Fonte: Autores.

\section{Beneficiamento das amostras}

As amostras naturais foram secas em estufa a $60^{\circ} \mathrm{C}$, em seguida beneficiadas, através de um britador de mandíbula da marca Marconi e posteriormente cominada em moinho de disco da marca Marconi, após moagem, as amostras foram passadas em peneira ABNT No200 (0,074mm).

\section{Análise granulométrica por difração a laser (AG)}

A difração a laser utiliza o método de dispersão de partículas em fase líquida associado com um processo de medida óptico através de difração a laser. Neste método, é combinada a relação proporcional entre a difração do laser e a concentração e tamanho de partículas. Para realização desta caracterização, as argilas bentoníticas naturais foram dispersas com hexametafosfato de sódio e agitadas por 20 minutos em agitador Hamilton Beach N5000 na velocidade de 17.000 rpm, em seguida ficou em repouso por $24 \mathrm{~h}$, após as $24 \mathrm{~h}$ em repouso as argilas foram agitadas novamente por cerca de 5 minutos sob a mesma velocidade, após agitação foi realizada a medida em modo úmido, até atingirem a concentração ideal que é de 170 unidades de difração/área de incidência.

\section{Análise química por fluorescência de raios X (EDX)}

As argilas bentoníticas foram passadas em peneira ABNT $\mathrm{N}^{\circ} 200(0,074 \mathrm{~mm})$ e submetidas à análise química por fluorescência de raios $\mathrm{X}$. $\mathrm{O}$ espectrômetro de fluorescência de raios $\mathrm{X}$ determina semi quantitativamente os elementos 
presentes em uma determinada amostra, através da aplicação de raios $\mathrm{X}$ na superfície da amostra e a posterior análise dos fluorescentes emitidos em equipamento EDX 720 da Shimadzu. A geração de raios-X é feita por meio de um tubo com alvo de $\mathrm{Rh}$.

\section{Difração de raios X (DRX)}

As argilas bentoníticas naturais e aditivadas com etileno glicol foram passadas em peneira ABNT n $200(0,074 \mathrm{~mm})$ e acondicionadas em porta amostra de Alumínio para análise por difração de raios X, em equipamento XRD 6000 da Shimadzu. A radiação utilizada foi a $\mathrm{K} \alpha$ do $\mathrm{Cu}(40 \mathrm{kV} / 30 \mathrm{~mA})$; a velocidade do goniômetro foi de $2^{\circ} / \mathrm{min}$ e passo de $0,02^{\circ}$; na faixa de $2^{\circ}$ a $60^{\circ}$.

\section{Análise térmica diferencial e termogravimétrica (DTA e TG)}

As análises termogravimétricas (TG) e termodiferenciais (DTA) são úteis para indicar as faixas de temperaturas onde ocorrem as perdas de massas e as temperaturas em que ocorrem transformações endotérmicas e exotérmicas. As curvas térmicas foram obtidas através de um sistema de Análises Térmicas Modelo RB-3000 da BP Engenharia Indústria e Comércio, com razão de aquecimento $12,5^{\circ} \mathrm{C} / \mathrm{min}$. A temperatura máxima para ambos os casos foi de $1000^{\circ} \mathrm{C}$ e o padrão utilizado na DTA foi o óxido de alumínio (A12O3) calcinado.

\section{Capacidade de troca de cátions (CTC)}

As CTC das amostras foram determinadas através do método de adsorção de azul de metileno, descrito em Ferreira et al; (1972). As etapas desse método são descritas a seguir:

Inicialmente, pesa-se $0,5 \mathrm{~g}$ de argila com granulometria inferior à peneira ${ }^{\circ} 325(0,044 \mathrm{~mm})$, coloca-se em um becker de $500 \mathrm{~mL}$ e mistura-se com $300 \mathrm{~mL}$ de água destilada;

Agita-se a suspensão e adiciona-se gota a gota solução $1 \mathrm{~N}$ de $\mathrm{Na} 2 \mathrm{CO} 3$ até o pH da suspensão atingir o valor de aproximadamente 9,0, a agitação deve continuar por $5 \mathrm{~min}$ para homogeneizar a mistura. Após a homogeneização, adiciona-se solução de $\mathrm{HCl} 1 \mathrm{~N}$, para reduzir o pH da suspensão até 3,5. Nesta condição de pH, supõe-se que todo azul de metileno esteja na forma mononuclear e apresente os melhores resultados;

Em seguida, titula-se a suspensão de argila com a solução padrão de azul de metileno da seguinte maneira: o titulante, solução de azul de metileno, é adicionada de $2 \mathrm{em} 2 \mathrm{~mL}$ e, após cada adição, a suspensão da argila é agitada continuamente a 5min, em seguida, objetivando averiguar se ponto de viragem foi alcançado, com uma baqueta de vidro, pinga-se uma gota da suspensão em papel de filtro Whatman $n^{\circ} 50$.

Para calcular a CTC das argilas determinadas pelo método de azul de metileno, deve-se utilizar a seguinte equação:

$\mathrm{CTC}=(\mathrm{V} * \mathrm{C} * 100) /($ massa da amostra seca $)$

onde: $\mathrm{V}=$ volume total $(\mathrm{mL})$ e $\mathrm{C}=$ concentração de azul de metileno.

Os resultados de CTC são apresentados em meq/100g de argila seca.

\section{Preparação das dispersões e estudo reológico}

As argilas naturais foram tratadas com solução concentrada de carbonato de sódio nas seguintes proporções: 75,100, 125 e 150 meq de $\mathrm{Na} 2 \mathrm{CO} 3 / 100 \mathrm{~g}$ de argila seca. Estes estudos preliminares indicaram que os melhores resultados para os 
parâmetros reológicos das argilas utilizadas nesta pesquisa, foram àqueles referentes à proporção de 125 meq de Na2CO3/100g de argila seca.

Os estudos reológicos para verificar a adequação dessas argilas em fluidos base água, foram realizados segundo norma Petrobrás EP-1EP-00011-A, 2011, sendo determinadas as viscosidades aparentes (VA) e plástica (VP) e volume do filtrado (VF).

\section{Resultados e Discussão}

\section{Análise granulométrica por difração a laser (AG)}

Na Figura 2 estão apresentadas as curvas de distribuição de tamanho de partículas das amostras estudadas.

Figura 2. Curvas de distribuição de tamanho de partículas das amostras estudadas.
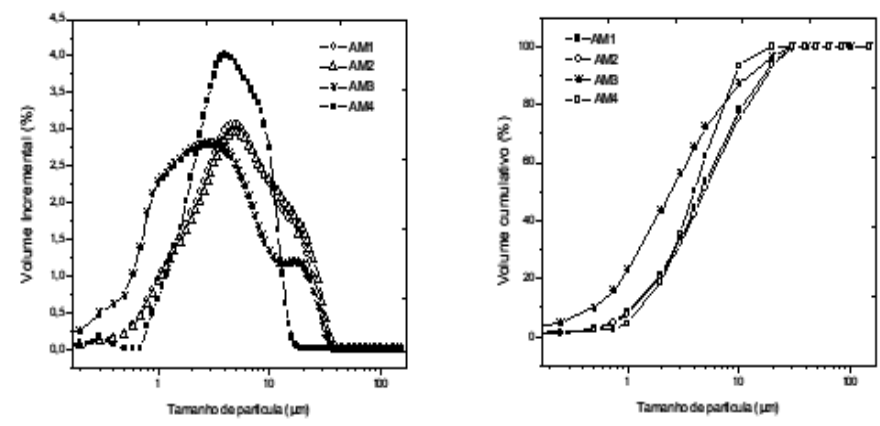

Fonte: Autores.

Na Tabela 1 encontram-se apresentados os valores da distribuição granulométrica das composições estudadas.

Tabela 1. Distribuição de tamanho de partículas das composições estudadas.

\begin{tabular}{l|cccc}
\hline \multicolumn{1}{c|}{ Amostra } & $\mathbf{x}<\mathbf{2 \mu m}$ & $\mathbf{2} \boldsymbol{\mu m}<\mathbf{x}<\mathbf{2 0} \boldsymbol{\mu m}$ & $\mathbf{x}>\mathbf{2 0} \boldsymbol{\mu m}$ & Diâmetro médio $(\boldsymbol{\mu m})$ \\
\hline Amostra 3 & 43,20 & 53,06 & 3,64 & 4,63 \\
\hline Amostra 4 & 18,80 & 81,02 & 0,0 & 4,71 \\
\hline
\end{tabular}

Fonte: Autores.

Observando os valores da Tabela 1, verifica-se que as amostras estudadas apresentaram diâmetro abaixo de $2 \mu \mathrm{m}$ correspondente a fração argila de 18 a $43 \%$ e diâmetro médio de partícula de 4,63 a 4,71 $\mu$ m.

Esses valores são similares aos encontrados nos estudos sobre argilas bentonitas Silva et al. (2015) e Pereira et al. (2015).

\section{Análise química por fluorescência de raios x (EDX)}

$\mathrm{Na}$ Tabela 2 estão apresentadas as composições químicas das amostras estudadas. 
Tabela 2. Composição química das argilas estudadas.

\begin{tabular}{ccccccccc}
\hline & \multicolumn{7}{c}{ Óxidos } \\
\cline { 2 - 9 } Amostra & $\mathbf{S i O}_{2}$ & $\mathrm{Al}_{2} \mathbf{O}_{3}$ & $\mathbf{M g O}$ & $\mathbf{F e}_{2} \mathbf{O}_{3}$ & $\mathbf{C a O}$ & $\mathbf{T i O}_{2}$ & Outros Óxidos & PR \\
& $(\%)$ & $(\%)$ & $(\%)$ & $(\%)$ & $(\%)$ & $(\%)$ & $(\%)$ & $(\%)$ \\
\hline AM3 & 55,4 & 18,1 & 3,2 & 8,7 & 2,4 & 1,0 & 2,2 & 9,0 \\
AM4 & 67,5 & 14,1 & 2,1 & 6,8 & 0,3 & 0,5 & 0,5 & 8,1 \\
\hline
\end{tabular}

Fonte: Autores.

Através da Tabela 2 observa-se que as composições analisadas apresentaram elevados teores de óxido de silício, variando de 55,4 a $67,5 \%$. Estes teores são originários do silício presente na camada tetraédrica do argilominerais como também dos minerais acessórios, sílica livre, mica e feldspato. Os valores de Al2O3 evidenciam uma variação de 14,1 a 18,1\% que definem a alumina presente na camada octaédrica e minerais acessórios.

Observou-se também que as amostras em estudo apresentaram teores elevados de óxido de ferro, sendo a amostra AM3 com 8,7\% e a amostra AM4 com 6,8\%, esses teores elevados nas amostras são oriundos da folha octaédrica dos argilominerais esmectíticos e dos minerais acessórios presentes com hematita, goetita e ilmenita. Estes teores são similares aos estudos de argilas bentoniticas do Estado da Paraíba (Pereira et al., 2015; Silva et al., 2015).

Os teores de $\mathrm{MgO}$ variaram entre 2,1\% (AM4) a 33,2\% (AM3), provavelmente da camada octaédrica da esmectita ou da dolomita ou carbonato de magnésio. Para os teores de $\mathrm{CaO}$ os valores variaram entre 0,3\% (AM2) a 2,4\% (AM1), provavelmente do mineral acessório calcita.

A perda ao rubro corresponde ao somatório da perda dos seguintes componentes: água livre e adsorvida, água coordenadas, intercalada entre as camadas e hidroxilas presente nos argilominerais, matéria orgânica e carbonatos existentes na argila. Observando a Tabela 2 conclui-se que os teores deste parâmetro variaram de 8,1\% a 9,0\%.

Os resultados encontrados neste trabalho foram semelhantes aos valores encontrados por Menezes et al., (2009), Pereira et al., (2015) e Silva et al., (2015), nos estudos preliminares sobre as argilas também pertencentes ao Estado da Paraíba.

\section{Difração de raios x (DRX)}

Na Figura 3 estão apresentados os difratogramas das amostras estudadas na forma natural e aditivada com etileno glicol.

Figura 3. Difratogramas das composições de amostras argilas estudadas.

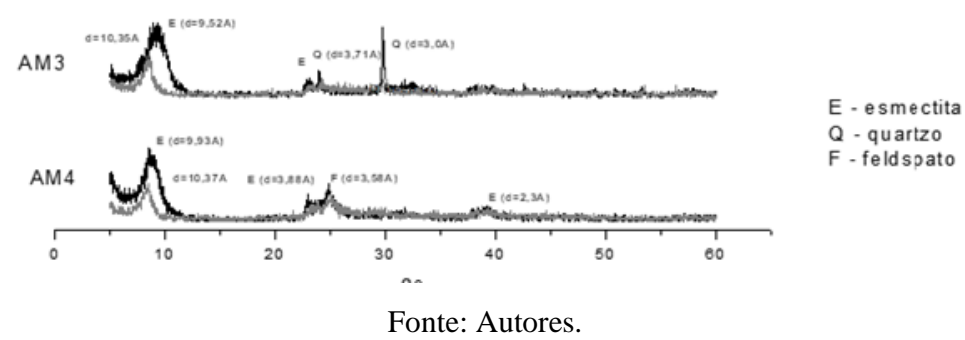

$\mathrm{Na}$ análise dos difratogramas da Figura 3, observa-se que todas as amostras estudadas apresentaram presença do 


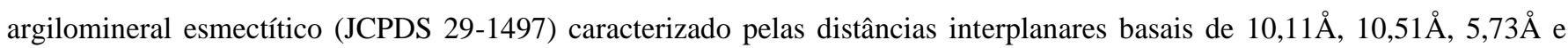

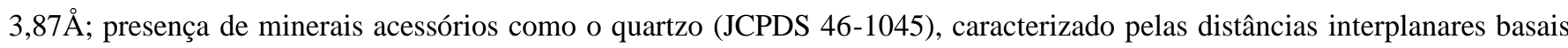

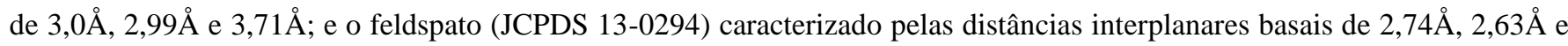
$3,58 \AA$.

A expansão das camadas interplanares basais, após adição do etilenoglicol, evidencia que as argilas estudadas pertencem ao grupo das esmectitas. Constata-se que as amostras estudadas neste trabalho apresentaram semelhança com outras amostras oriundas do Estado da Paraíba (Menezes et al., 2009; Pereira et al., 2015; Santos, 1992; Silva et al., 2015).

\section{Análise térmica diferencial e termogravimétrica (DTA E TG)}

Na Figura 4 estão apresentadas as curvas de análises termogravimétricas e térmicas diferenciais das argilas estudadas.

Figura 4. Curvas de análises termogravimétricas e térmicas diferenciais das argilas.
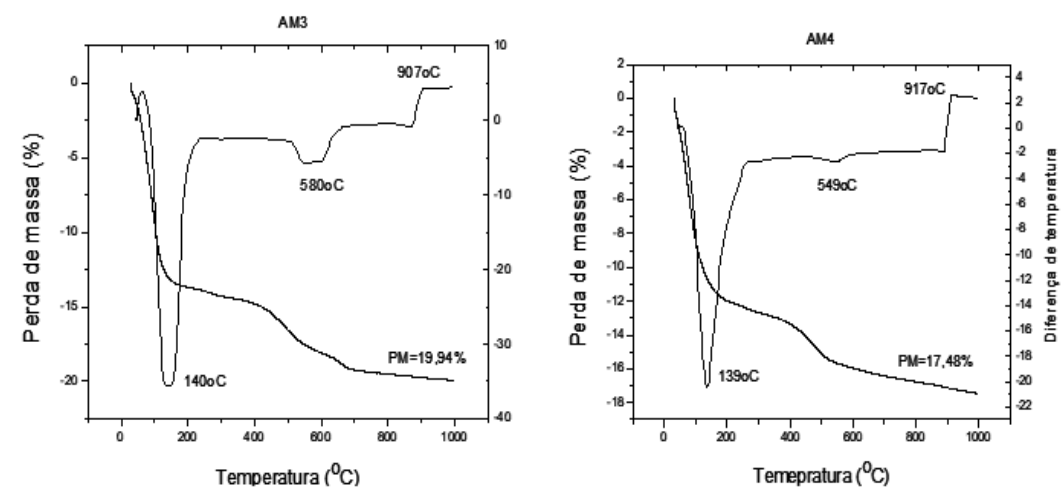

Fonte: Autores.

Analisando as curvas dos termogramas das amostras estudadas (Figura 4), pode-se observar as seguintes transformações térmicas: grande pico endotérmico a aproximadamente $140^{\circ} \mathrm{C}$, caracterizando a presença de água livre e adsorvida e um ombro aproximadamente $160^{\circ} \mathrm{C}$, característico da presença de água intercaladas entre as camadas; pico endotérmico em torno de $550^{\circ} \mathrm{C}$ caracterizando a desidroxilação da esmectita.

Comparando os resultados dos termogramas com os valores encontrados por Silva et al., (2013) para as argilas de Pedra Lavrada/PB, bem como Menezes et al. (2009) para argilas de Cubati/PB, e por Pereira et al., (2014) e Pereira et al., (2015) Sossego/PB, observa-se que são similares, evidenciando um comportamento típico das argilas bentoníticas oriundas do Estado da Paraíba.

\section{Capacidade de troca de cátions (CTC)}

Na Tabela 3 estão apresentados os resultados da capacidade de troca de cátions, determinadas através do método de azul de metileno das composições estudadas. 
Tabela 3. Capacidade de troca de cátions das composições estudadas.

\begin{tabular}{c|c}
\hline Amostra & CTC (meq/100g argila seca) \\
\hline AM3 & $\mathbf{8 8}$ \\
\hline AM4 & 56 \\
\hline
\end{tabular}

Fonte: Autores.

Analisando os valores da Tabela 3, verifica-se que a amostra AM4 apresentou valor de CTC inferior a faixa dos argilominerais do grupo das esmectitas (80 a 150 meq/100g), enquanto a amostra AM3 do Município de Boa Vista/PB, apresentou CTC igual a $88 \mathrm{meq} / 100 \mathrm{~g}$ de argila seca.

\section{Propriedas reológicas}

Na Tabela 4 estão apresentados os resultados das propriedades reológicas: Viscosidade Aparente(VA), Viscosidade Plásticas(VP) e Volume de Filtrado(VF) segundo Normas da Petrobrás composições estudadas.

Tabela 4. Propriedades reológicas.

\begin{tabular}{c|ccc}
\hline Amostra & VA (cP) & VP (cP) & VF (mL) \\
\hline AM3 & 17 & $\mathbf{6}$ & $\mathbf{1 7}$ \\
\hline AM4 & $\mathbf{1 6}$ & $\mathbf{7}$ & $\mathbf{1 8}$ \\
\hline
\end{tabular}

Fonte: Autores.

Analisando a Tabela 4, podemos observar que os resultados reológicos da amostra AM3 e AM4, atingiram as especificações estabelecidas pela Petrobrás EP-1EP-00011-A da Petrobrás, 2011, uma vez que a VA foram superiores 15,0cP, VP maior do $5 \mathrm{cP}$ e o volume de filtrado menores de $18,0 \mathrm{~mL}$.

\section{Conclusão}

Com o objetivo de estudar e comparar as propriedades reológicas de duas variedades de argilas esmectitícas dos Municípios de Boa Vista do Estado da Paraíba como viscosificante para uso em fluidos de perfuração de poços de petróleo base água foi possível concluir que:

Após a caracterização observou-se que ambas as argilas apresentam propriedades de argilas esmectitas;

A argila denominada de Chocolate e Bofe apresentam valores de VA, VP e VF dentro das especificações da Petrobrás para uso em fluidos de perfuração base água, sendo adequada para o uso nos fluidos.

Os resultados mostraram a importância da caracterização e do estudo reológico das argilas esmectitas, pois através dele conseguimos compreender suas propriedades e suas aplicações.

Sugerimos o estudo comparativo entre diferentes tipos de argilas não analisadas, proporcionando assim, aumento do banco de dados científico e embasamento para futuras aplicações práticas. 


\section{Referências}

Abu-Jdayil, B. (2011). Rheology of sodium and calcium bentonite-water dispersions: Effect of electrolytes and aging time. International Journal of Mineral Processing. 98, 208-213.

Araújo, E. M., Melo, T. J. A., Santana, L. N. L., Neves, G. A., Ferreira, H. C., Lira, H. L., Carvalho, L. H., A'vila Jr., M. M., Pontes, M. K. G., Araújo, I. S. (2004). The influence of organobentonite Clay on the processing and mechanical properties of nylon 6 and polystyrene composites. Materials Science and Engineering. B 112, 175-178.

Bergaya, F.; Theng, B. K. G.; Lagaly, G. (2006). Developments in Clay Science, ed. Elsevier. Chapter 1, p. 1 - 18.

Cara, S., Carcangiu, G., Padalino, G., Palomba, M., Tamanini, M. (2000). The bentonite in pelotherapy: chemical, mineralogical and technological properties of materials from Sardinia deposits (Italy). Applied Clay Science. 16, 117-124.

Corrales, T., Larraza, I., Catalina, F., Portoles, T., Ramírez-Santillan, C., Matesanz, M., Abrusci, C. (2012). In vitro biocompatibility and antimicrobial activity of poly ( $\varepsilon$ - caprolactone)/montmorillonite nanocomposites. Biomacromolecules. 13, 4247-4256.

Grimre \& Güvenn. (1998). Bentonites, Geol-ogy, Mineralogy, Properties and Uses. Developmentsin Sedimentology 24, Elsevier, Amsterdam, Nether-lands, $256 \mathrm{p}$.

Lee, Y. H., Kuo, T. F., Chen, B. Y., Feng, Y. K., Wen, Y. R., Lin, W.C., Lin, F. H. (2005). Toxicity assessment of montmorillonite as a drug carrier for pharmaceutical applications: yeast and rats model. Biomed.Eng. Appl. BasisCommun. 17, 72-78.

Liu, Q., Liu, Y., Xiang, S., Mo, X., Su, S., Zhang, I. (2011). Apoptosis and cytotoxity of oligo (styrene-co-acrylonitrile) modified montmorilonite. APPL. Clay Sci. 51, 214-219.

Lima, T.M.; Neves, C. A. R. (2014). Departamento Nacional de Produção Mineral, DNPM. Sumário Brasileiro de Mineração, v. 34 , p. 141.

Menezes, R. R.; Campos, L. F. A.; Ferreira, H. S.; Marques, L. N.; Neves, G. A.; Ferreira, H.C. (2009). Estudo do comportamento reológico das argilas bentoníticas de Cubatí, Paraíba, Brasil. Cerâmica, v.55, p. 349 - 355.

Murray, H. H. (2007). Applied Clay Mineralogy. Developments in Clay Science, $1^{\mathrm{a} E d .}$

Nascimento, R. C. A. M.; Amorim, L. V.; Santana, L. N. L. (2010). Desenvolvimento de fluidos aquosos com bentonita para perfuração de poços de Petróleo onshore. Cerâmica, v. 56, p. 179 - 187.

Oliveira, F. das C., Sousa, R. M. L., Silva, P. F.., Lopes, P. D., Leite, R. C., Salgado, M. de F. ., Paz, G. M. . da ., \& Soares, R. A. L. . (2021). Caracterização de Materiais: A Termodinâmica e suas aplicações no estudo da análise térmica de argilas. Research, Society and Development, $10(2)$, e6510212284. https://doi.org/10.33448/rsd-v10i2.12284.

Petrobrás. (2011). Ensaio de viscosificante para fluidos base água na exploração e produção de petróleo. Código:EP-1EP-00011-A.

Pereira, I. D. S.; Lisboa, V. N. F.; Silva. I. A.; Figueirêdo. J. M. R.; Neves, G. A.; Menezes. R. R. (2015). Bentonite Clays Characterization in the Town of Sossego - Paraiba State. Materials Science Forum. v. 820, p. 65 - 70.

Pereira, I. D. S.; Silva, I. A.; Cartaxo, J. M.; Menezes, R. R.; Santana, L. N. L.; Neves, G. A.; Ferreira, H. C. (2014a) Estudos de caracterização dos novos depósitos de argilas esmectitas do munícipio de Sossego, PB. Cerâmica, v. 60, p. 223 - 230.

Rodrigues, M. de F. da S., Veldhuis, R., Gomes, L. K. M., Menezes, J. M. C., Teixeira, R. N. P., \& Silva, J. H. da. (2020). Avaliação da capacidade retentiva de metais tóxicos provenientes de indústrias de galvanoplastia utilizando matrizes cerâmicas. Research, Society and Development, 9(7), e679974616. https://doi.org/10.33448/rsd-v9i7.4616.

Santos, S. P. (1992). Ciência e tecnologia de argilas. Vol. 1, 2a edição. São Paulo: Editora Edgard BlücherLtda, 1989. ISBN-10: 8521201982.

Silva, I. A.; Pereira, I. D. S.; Cavalcanti, W. S.; Sousa, F. K. A.; Nevesa, G. A; Ferreira, H. C. (2015). Study of the Characterization of Smectitic Clays from a Town in the State of Paraíba. Materials Science Forum, v. 820, p. 51 - 55.

Silva, M. de L.., \& Pereira, F. M. M. (2021). Caracterização de argilas provenientes de indústria de cerâmica vermelha do Município do Crato - Ceará, Brasil. Research, Society and Development, 10(10), e448101018392. https://doi.org/10.33448/rsd-v10i10.18392.

Thomas, J. E. (2001). Fundamentos de engenharia de petróleo, Ed. Interciência, Rio de Janeiro, RJ.

Toor, M., Jin, B. (2012). Adsorption characteristics, isotherm, kinetics, and diffusion of modified natural bentonite for removing diazo dye. Chem. Eng. J. 187, 79-88.

Vieira, M. N. A.., Alves, C. N., Ferreira, V. dos S., \& Barbosa, C. N. L. (2021). Modelagem de propriedades geológico-geotécnicas em solos de Belém do Pará. Research, Society and Development, 10(2), e40810212664. https://doi.org/10.33448/rsd-v10i2.12664

Viseras, C., Cerezo, P., Sanchez, R., Salcedo, I., Aguzzi, C. (2010). Current challenges in Clay minerals for drug delivery. Applied Clay Science 48, 291-295. 